\title{
A non-randomised controlled trial of the R\&R2MHP cognitive skills program in high risk male offenders with severe mental illness
}

Vivienne C-Y Yip ${ }^{1,2}$, Gisli H Gudjonsson 2,3 ${ }^{2, \text { Derek Perkins }}{ }^{2}$, Amie Doidge ${ }^{2}$, Gareth Hopkin ${ }^{2}$ and Susan Young ${ }^{1,2^{*}}$

\begin{abstract}
Background: The growing popularity of offending behavior programs has led to the interest of whether such programs are effective with mentally disordered offenders. This study aimed to evaluate the effectiveness of the Reasoning and Rehabilitation program adapted for offenders with severe mental illness (R\&R2 MHP).

Methods: A sample of 59 adult high risk males detained in a high secure hospital completed questionnaires at baseline and post treatment to assess violent attitudes, anger, coping processes and social problem-solving. An informant measure of social and psychological functioning, including disruptive behavior, was completed by staff at the same time. The data of 30 patients who participated in the group condition were compared using intention to treat analysis with 29 controls who received treatment as usual.
\end{abstract}

Results: $80 \%$ of group participants completed the program. In contrast to controls, significant medium-large treatment effects were found at outcome on self-reported measures of violent attitudes, social problem-solving and coping processes. Improvements were endorsed by informant ratings of disruptive behavior, social and psychological functioning.

Conclusions: The R\&R2MHP had a comparatively low dropout rate and was effective in a sample of high risk mentally disordered offenders requiring detention in high security. Future research should use a randomized controlled design.

Trial registration: Current Controlled Trials ACTRN12613000216718.

Keywords: Mentally disordered offenders, Treatment outcome, Reasoning \& Rehabilitation, Cognitive skills

\section{Background}

Individuals with severe mental illness (SMI) are at an increased risk of committing violent crimes. This is a robust finding supported by a variety of studies using a range of different methodologies including: population cohort studies [1-4]; community prevalence studies [5,6] and retrospective studies $[7,8]$. This is also evidenced by the high occupation of forensic inpatient beds by those who have SMI [9]. To alleviate this pressure and as prison and hospital populations rise, there is an increased demand for evidence-based treatments designed to reduce antisocial behaviors in those with SMI.

\footnotetext{
* Correspondence: susan.young@kcl.ac.uk

${ }^{1}$ Department of Forensic and Neurodevelopmental Sciences, PO23, King's

College London, Institute of Psychiatry, De Crespigny Park, London SE5 8AF, UK ${ }^{2}$ Broadmoor Hospital, Crowthorne, UK

Full list of author information is available at the end of the article
}

Research indicates that predictors of recidivism are similar for offenders with and without mental disorder $[10,11]$. These shared predictors include: maladaptive beliefs and attitudes supporting a criminal lifestyle [12,13] and poor social problem-solving ability [14-17]. Indeed, pro-criminal thinking has emerged as the strongest predictor for offending behavior [18-20]; others include institutional violence [21], poor psychosocial functioning and treatment engagement [22]. The association with anger is less clear; some research has reported it is a greater predictor of aggressive behavior than pro-criminal attitudes whereas other research suggests it is not necessarily associated with aggressive behavior [23,24].

Detainment is the most conventional method of disposal for those who commit severe criminal offences, yet there is paucity of research reviewing how offenders 
cope with their detainment and how it influences reoffending after release from prison. Zamble and Porporino [25] propose that an individual's ability to cope with life circumstances is a critical factor for future adaptation. In their investigation of coping behaviors in imprisoned offenders, they found that offenders exhibited poor coping behaviors which maintained criminal behavior and moreover led to recidivism.

\section{Treating high risk offenders}

Mentally disordered offenders (MDOs) with complex clinical needs and severe aggression are commonly detained in high secure hospitals. They are considered 'high risk' as they pose a grave and immediate danger to the public (Department of Health, 2006). Compared with those from low and medium secure hospitals, these patients have a diverse and complex range of psychiatric and criminogenic needs, often presenting with high rates of comorbidity and difficulty engaging with their clinical care [26,27].

Martinson [28] sparked debate after claiming that nothing worked in offender rehabilitation. This led to closer investigations of the efficacy of offender rehabilitation programs and has subsequently contributed to an amassing wealth of 'what works' research [29], which indicates that treatments targeted towards rehabilitating offenders are effective. Blud et al. [30] suggested that reducing recidivism was possible if interventions were appropriately designed, focused towards aims and delivered in a systematic manner.

Since then, a growing interest has developed in the effectiveness of Offending Behavior Programs (OBPs), the most common being the 22-session Enhanced Thinking Skills and the 36-session Reasoning and Rehabilitation $[R \& R]$ programs [31,32]. Both these OBPs employ a cognitive-behavioral paradigm which has been shown to be effective for reducing recidivism rates in juveniles and adult offenders $[33,34]$. However, these OBPs have not been designed to meet the complex needs of MDOs. Whilst a treatment effect was reported for clinical outcomes in a randomised control trial of the original $R \& R$ program, there was a 50\% attrition rate of those allocated to the treatment condition [35]. This is important from a risk perspective, as research suggests that recidivism rates are elevated amongst non-completers [36-38]. Antisocial personality disorder, recent violence and psychopathy have been found to predict dropout [35].

High rates of treatment dropout call into question whether OBPs with a general offending focus are relevant and responsive to the needs of MDOs. The Risk Need Responsivity model suggests that structured and targeted programs should be delivered with the aim to match content and pace of treatment with specific offender characteristics [39]. Consistent with this theoretical paradigm, $R \& R$ has been adapted to meet the needs of MDOs (R\&R2MHP)
[40]. Firstly, the program duration was reduced from 36 to 16 sessions but supplemented by individual sessions provided by a mentor. The mentor role was introduced to improve treatment completion [41] and support the patient to rehearse and consolidate newly acquired skills and transfer them into daily activities. Secondly by inclusion of a module that addresses the executive dysfunction (such as attention and memory problems, poor organizational and planning skills) commonly experienced by many patients with SMI and that may interfere with their ability to effectively engage in OBPs [42].

Research has indicated that R\&R2MHP is a feasible and effective treatment for MDOs [43,44]. A controlled multi-site study of patients detained in medium and low secure services reported a completion rate of $78 \%$ and positive clinical changes on self-reported measures of rational problem-solving, anger and attitudes towards violence. Improvement was supported by informant ratings of social and psychological functioning.

An evaluation of a similar program (R\&R2 for ADHD Youths and Adults) in MDOs with a primary diagnosis of personality disorder detained in a high secure service has also shown a positive treatment effect, together with a $76 \%$ program completion rate [45]. However feasibility and effectiveness of R\&R2MHP has yet to be investigated in high risk MDOs with a primary diagnosis of SMI. Furthermore, the treatment effect for improving coping processes in high risk MDOs has yet to be established. This study therefore evaluated the R\&R2MHP in such patients using a quasi-experimental design comparing group participants with waiting list controls. The following research questions were investigated:

\section{How successful is the program in terms of retention of participants in a high secure setting?}

Consistent with the findings of Rees-Jones et al. [43], it was hypothesised that the completion rate would be greater than the $50 \%$ reported by Cullen et al. [35] in their study using the original 36 session R\&R program.

\section{Is the program effective in patients detained in a high secure setting?}

Compared to controls, it was hypothesised that group participants would show significant improvements in violent attitudes, anger, coping processes, social problemsolving, disruptive behavior and social functioning.

\section{Methods}

\section{Design and participants}

The quasi-experimental controlled study involved the participation of 59 male patients with SMI detained under the U.K. Mental Health Act (1983) in a high secure hospital 
setting. Thirty patients participated in the treatment condition (R\&R2MHP). A total of five groups were delivered for this study. Their data were compared with that of 29 controls who received treatment as usual (TAU) (see Figure 1).

All participants were referred by their clinical team to attend the OBP. Inclusion criteria were age 18-65, a history of SMI (e.g. schizophrenia, schizoaffective disorder, bipolar disorder), a history of violent or antisocial behavior, no previous experience of participating in R\&R, proficiency in English language. Exclusion criteria included intellectual disability, patients who were mentally unstable and/or who posed a risk of violence to the researcher.

\section{Intervention}

R\&R2MHP [40] is a manualised cognitive-behavioral intervention developed for antisocial youths and adults with mental health problems. It consists of 16, 90-minute, sessions which run on a weekly basis. It is a revised version of the original 36-session $R \& R$ program and aims to reduce anti-social behavior and attitudes and improve pro-social thinking, cognitive and problem-solving skills. The program employs a variety of methods to engage individuals such as individual group exercises, audiovisual material and workbooks which include homework assignments. The program consists of 5 treatment modules: (1) a neurocognitive model which introduces techniques to increase attentional control, impulse control, memory and constructive planning; (2) a problem-solving module which encourages problem identification, generation of multiple alternative solutions and consequential thinking; (3) an emotional control module which involves management of anxiety, anger and conflict; (4) a social skills module which aims to increase awareness of the thoughts and feelings of others; and (5) a critical reasoning module which aims to develop skills in the assessment and evaluation of information. The R\&R2MHP program combines group and individual treatment. Individual treatment incorporates the use of a mentoring paradigm, whereby a mentor provides coaching sessions outside of group sessions. Their purpose is to facilitate the transfer of skills learned in group sessions into daily activities. Mentors are provided with written guidance on how to structure individual sessions. R\&R2 MHP facilitators were provided with training in how to deliver the program. Program integrity and consistency was ensured through (1) establishing a steering committee, where regular meetings were held and supervision was provided by SY (the program author); (2) random observations of group sessions by one of the program authors (SY); and (3) group supervision meetings of facilitators to prepare for sessions, process and discuss sessions that had been delivered and (4) regular meetings and supervision between program facilitators and mentors. Furthermore, R\&R2MHP maximised program integrity by fostering consistency through its structured, manualised design.

\section{Treatment completion}

A cut-off of $\geq 12$ sessions was used to classify patients as completers, following the recommendation made by Cullen et al. [46] and representing $80 \%$ attendance of the programme.

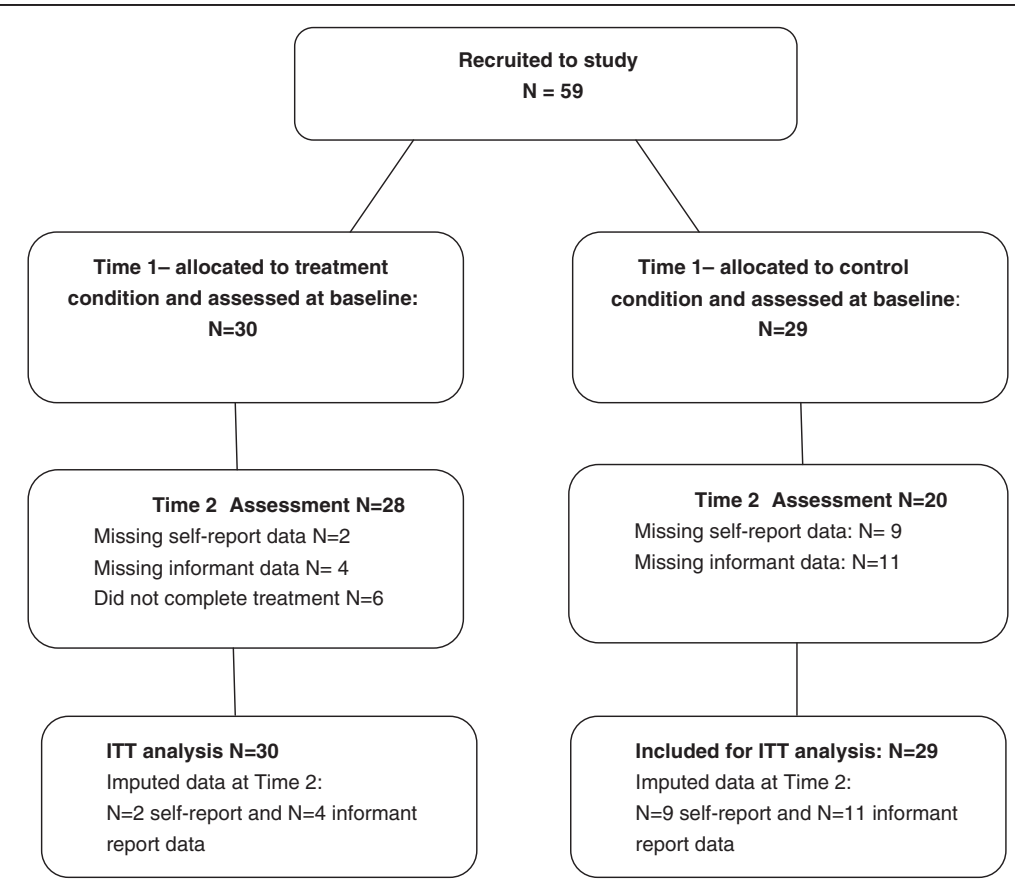

Figure 1 Flowchart of patient participation. 


\section{Treatment as usual}

Control participants were not asked to refrain from attending interventions considered to be part of their usual treatment. However they were not permitted to attend the R\&R2MHP program or similar cognitive skills interventions during the course of the study. Common psychosocial interventions included group and individual occupational, psycho-educational and cognitive-behavioral interventions for psychosis, personality disorder, violence, sex offending, substance misuse and relapse prevention.

\section{Measures}

\section{Baseline assessments}

Demographic information, diagnosis, offence and admission history were obtained from clinical file reviews at the start of the study. In addition participants completed the Psychotic Symptoms Rating Scale (PSYRATS) [47] to assess severity of psychotic symptoms and the structured clinical interview for DSM-III-R personality disorders (SCID II) [48] to assess antisocial personality characteristics.

\section{Outcome measures}

The following measures were administered at baseline (Time 1) and repeated 16 weeks later post group (Time 2) to assess the primary (violent attitudes) and secondary outcomes (coping skills, social problem-solving, reaction to provocation, disruptive behavior and social functioning). The primary outcome measure was determined prior to commencement of the study. All measures were self-rated, except for the informant-rated Disruptive Behavior and Social Problem Scale (DBSP). The same informant (a member of the clinical team) completed the DBSP at Time 1 and Time 2.

1. Maudsley Violence Questionnaire (MVQ) [49] is a 56-item self-reported measure of cognitive style in relation to violent attitudes. It has two constructs: machismo (42 items which assess the extent to which the individual supports stereotypes of males as being dominant and tough) and acceptance (14 items which assess the acceptance of violence). A total score can be obtained by summing the scale scores (score range $0-56)$. High internal-consistency reliability for both constructs was estimated by Cronbach's alpha (ranging between 0.74 and 0.91) in a male student sample [49] and it has specified differences between mentally disordered offenders [43,44].

2. Novaco Anger Scale - Provocation Inventory: Reaction to Provocation/Personal Affect Questionnaire (NAS-PI) [50] is a 48-item self-reported measure that assesses cognitive, arousal and behavioral domains of anger experience. Each item is rated on a 3-point Likert scale with higher scores indicating greater endorsement of anger (scores range between 16-48 for each domain); a total score can also be obtained by summing the domain scores (score range 48-144). The NAS-PI has good test-retest reliability (Cronbach's alphas ranged from 0.78 to 0.91 ), concurrent validity, and the NAS total score has excellent internal-consistency (Cronbach's alpha 0.92) [51,52]. It has been effective in predicting violence in MDOs [53] and discriminating between aggressive patients and non-clinical controls [54].

3. Ways of Coping Scale (WAYS) [55] is a 66-item self-reported measure of coping processes with responses. Participants are asked to recall a stressful situation that they have experienced in the past week. They are informed that this may be a situation that was difficult or troubling for them, either because they felt distressed about what happened, or because they had to use considerable effort to deal with the situation. They are told that the situation may have involved their family, work, friends or something else important to them. They are then asked to rate how often they used suggested behaviors to cope with that particular stressor on a 4-point Likert scale. The WAYS includes eight constructs, each with good internal consistency estimated by Conbach's alpha as follows: (1) confrontive coping, 0.70; (2) distancing, 0.61; (3) self-controlling, 0.70; (4) social support, 0.76; (5) accepting responsibility, 0.66; (6) escape avoidance, 0.72; (7) planful problem-solving, 0.68 ; and (8) positive reappraisal, 0.68 [56]. In addition a total score can be obtained by summing the scale scores. The higher the score, the more effort the person applies to the coping process.

4. Social Problem-Solving Inventory-Revised: Short (SPSI-R: S) [14] is a 25-item self-reported questionnaire with responses rated on a 5-point Likert scale that assesses people's ability to solve problems in everyday life. It consists of five subscales, two measuring problem-solving orientation (positive and negative) and three assessing problem-solving style (rational problem-solving, impulsivity/ carelessness and avoidant). Scores range between 0-20 for each domain. An adjusted total score was obtained (score range 0-20) with higher scores reflecting better social problem-solving ability. This measure is reported to have high test-retest reliability (Cronbach's alphas ranged from 0.68 to 0.91 ) and internal consistency (Cronbach's alphas ranged from 0.69 to 0.95 ) [57].

5. Disruptive Behavior and Social Problems Scale (DBSP) [58] is an informant-rated questionnaire consisting of 14 statements rated on a 7-point Likert scale relating to patient's behavior and social interactions. The scale consists of two factors, (1) 
disruptive behavior (score range 8-56), and (2) social and psychological functioning (score range 6-42). Higher scores indicate a greater degree of problems. Both factors have excellent or good internal consistency (Cronbach's alpha 0.92 and 0.84 respectively).

\section{Procedure}

Approval of the research project was given by Ealing and West London Research Ethics Committee. Patients meeting inclusion criteria and who were considered to be mentally stable and suitable for this intervention were referred by their clinical team. Referred patients were approached and invited to participate in the study. The treatment was not mandatory. A waiting-list controlled design was utilised and group allocation was determined by the order of referral. Once group capacity had been reached, the remaining patients were kept on a waiting-list for the following group. After providing informed consent, participants completed the self-reported measures at baseline (Time 1) and data were obtained from clinical file reviews. The DBSP was completed by a member of the clinical team who knew the patient well (most commonly the primary nurse). To reduce inter-rater differences, the same informant completed the questionnaire at both Time 1 and Time 2. Outcome measures were repeated again on completion of the group (Time 2). The timing between assessments was generally the same (16-18 weeks) for the R\&R2MHP and TAU conditions. A total of 5 groups, each with 5-8 participants were delivered weekly. In addition group participants met with their mentor (a member of the clinical team, most commonly a health care or psychology assistant) between sessions. Session logs were completed to record group attendance. Researchers involved with data collection were not involved in the delivery of the treatment. Data were compiled centrally, input and scored using the Statistical Program for Social Sciences (SPSS) database.

\section{Statistical analysis}

Descriptive statistics summarized demographics, clinical and forensic baseline characteristics. Independent-samples $\mathrm{t}$ tests were used to examine group differences at Time 1 (see Tables 1 and 2). Unadjusted mean scores and standard deviations on each of the outcome measures are provided in Table 3. A conservative intention to treat (ITT) analysis was used to assess outcome with missing data imputed by 'last observation carried forward'. Figure 1 provides a flowchart of patient participation and shows that data were imputed at outcome for two treatment participants (7\% of the treatment condition) and 9 control participants (31\% of the control condition). This design (ITT) was adopted as this analysis is based on initial treatment intent rather than the treatment which was eventually administered [59]. Demographic and total score differences
Table 1 Participant characteristics comparing group participants (R\&R2MHP) and controls (TAU) at baseline (Time 1)

\begin{tabular}{|c|c|c|c|c|c|}
\hline & \multicolumn{2}{|c|}{ R\&R2MHP group } & \multicolumn{2}{|c|}{ TAU group } & \multirow[b]{2}{*}{ t-value } \\
\hline & $\mathrm{N}$ & $M(S D)$ & $\mathrm{N}$ & $M(S D)$ & \\
\hline \multirow[t]{2}{*}{ Age } & 30 & $37.93(10.10)$ & 29 & $38.72(9.70)$ & -0.31 \\
\hline & & Range 22-63 & & Range 20-66 & \\
\hline \multirow{2}{*}{$\begin{array}{l}\text { Number of previous } \\
\text { admissions }\end{array}$} & 30 & $4.07(4.56)$ & 29 & $3.24(4.64)$ & 0.69 \\
\hline & & Range 0-15 & & Range 0-20 & \\
\hline \multirow{2}{*}{$\begin{array}{l}\text { Number of previous } \\
\text { convictions }\end{array}$} & 30 & $10.47(14.45)$ & 29 & $14.00(20.30)$ & -0.77 \\
\hline & & Range 0-65 & & Range 0-100 & \\
\hline PSYRATS total & 30 & $7.87(14.00)$ & 29 & $2.79(10.50)$ & 1.57 \\
\hline SCID-II total & 30 & $30.27(12.48)$ & 29 & $34.59(9.78)$ & -1.48 \\
\hline MVQ total & 30 & $15.83(12.02)$ & 29 & $18.14(13.45)$ & -0.69 \\
\hline NAS-PI total & 30 & 77.27 (16.78) & 29 & $79.24(15.74)$ & -0.47 \\
\hline WAYS total & 30 & $46.90(22.42)$ & 29 & $42.82(24.86)$ & 0.66 \\
\hline SPSI total & 30 & 12.39 (3.36) & 29 & $11.57(2.49)$ & 1.07 \\
\hline DBSP total & 26 & 35.59 (16.12) & 27 & $39.00(12.51)$ & -0.84 \\
\hline
\end{tabular}

between the two groups at Time 1 were not significant for baseline and outcome measures, nevertheless in order to minimize error variance a two-tailed analysis of covariance (ANCOVA) was administered for each of the dependent variables measuring differences between the conditions in time using adjusted mean scores and standard deviations (see Table 3). The baseline Time 1 scores therefore served as covariates for the dependent Time 2 variables. The effect size was analyzed using Cohen's $d$ for efficacy measures.

In addition a post-hoc per protocol (PP) analysis was performed on the subgroup of participants for whom full data at Times 1 and 2 were available.

\section{Power calculation}

Sample size was determined by a power calculation based on data obtained from a pilot study conducted by Young, Chick and Gudjonsson [44]. Calculations at 80\% power with an alpha level of 0.05 suggested that 35 participants per group will be needed in order to detect a difference in the primary outcome measure with an effect size of 0.42 (pre-treatment mean $15.95(\mathrm{SD}=10.83)$ and post-treatment mean $11.36(\mathrm{SD}=10.53)$.

\section{Results}

Baseline characteristics

All participants had a history of severe mental illness, most commonly psychotic disorders, and violent offending including with index offences of homicide (37\%), sexual violence (24\%) and assault (39\%). Independent $t$-tests showed that there were no significant differences between 
Table 2 Participant characteristics comparing group completers with non-completers at baseline (Time 1)

\begin{tabular}{|c|c|c|c|c|c|}
\hline & \multicolumn{2}{|c|}{ R\&R2MHP completers } & \multicolumn{2}{|c|}{ R\&R2MHP Non-completers } & \multirow[b]{2}{*}{ t-value } \\
\hline & $\mathbf{N}$ & $M(S D)$ & $\mathbf{N}$ & $M(S D)$ & \\
\hline \multirow[t]{2}{*}{ Age } & 24 & $36.67(9.42)$ & 6 & $43.00(12.05)$ & -1.40 \\
\hline & & Range $=22-59$ & & Range $=31-63$ & \\
\hline \multirow[t]{2}{*}{ Number of previous admissions } & 24 & $4.08(4.40)$ & 6 & $4.00(5.62)$ & 0.04 \\
\hline & & Range $=0-14$ & & Range $=0-15$ & \\
\hline \multirow[t]{2}{*}{ Number of previous convictions } & 24 & $10.88(15.16)$ & 6 & $8.83(12.25)$ & 0.31 \\
\hline & & Range $=0-65$ & & Range $=0-33$ & \\
\hline PSYRATS total & 24 & $7.83(14.58)$ & 6 & $8.00(12.65)$ & -0.03 \\
\hline SCID-II total & 24 & $31.04(12.08)$ & 6 & $27.17(14.76)$ & 0.67 \\
\hline MVQ total & 24 & $14.29(11.20)$ & 6 & $22.00(14.30)$ & -1.43 \\
\hline NAS-PI total & 24 & $77.04(16.24)$ & 6 & $78.17(20.48)$ & -0.14 \\
\hline WAYS total & 24 & $46.79(20.96)$ & 6 & $47.33(29.94)$ & -0.05 \\
\hline SPSI total & 24 & $12.65(3.07)$ & 6 & $11.37(4.55)$ & 0.83 \\
\hline DBSP total & 21 & $32.67(13.50)$ & 5 & $48.40(21.50)$ & $-2.09^{*}$ \\
\hline
\end{tabular}

${ }^{*} \mathrm{p}<.05$.

the R\&R2MHP and TAU groups for age, previous number of admissions and convictions, current psychotic symptoms (PSYRATS), antisocial personality traits (SCID-II), and the total scores of outcome measures administered pre-treatment (see Table 1).

\section{Program completion rate}

Of the 30 participants who started the R\&R2MHP program, 24 completed the program giving a group completion rate of $80 \%$. Group completers attended a mean of 14 sessions (SD 1.38; range 12-16) and the non-completers attended a mean of 7 sessions (SD 3.73; range 2-11).

Table 2 shows that there were no significant differences between completers and non-completers in age, number of previous convictions and admissions. Nor were significant differences found between R\&R2MHP completers and non-completers on total scores of self-reported outcome measures administered at Time 1. However the non-completers were rated by informants to be significantly more behaviorally disturbed on the DBSP.

\section{Post-treatment outcome with ITT analysis}

Table 3 presents unadjusted means and standard deviations for each of the outcome measures at baseline (Time 1) and outcome (Time 2) for both R\&R2MHP and TAU. Results showed that being in the treatment group significantly reduced violent attitudes with medium effect for the Total MVQ score and Acceptance of Violence scale, and with small effect for the Machismo scale. No significant differences were found on NAS-PI scores assessing anger reactions to provocation.

All treatment effects for the WAYS coping processes were large. A significant improvement in effortful coping was found for the Total score, together with applying strategies relating to confrontive, distancing, applying selfcontrol, seeking social support, accepting responsibility, escape-avoidance, planful problem-solving and positive reappraisal.

For social problem-solving, significant treatment effects were found for developing a more positive problem-solving orientation (large effect) and for engaging in a more rational problem-solving style (large effect) and avoidance problem-solving style (small effect). No significant differences were found between groups for the SPSI-RS Total score, negative problem solving orientation or impulsive/ carelessness style.

Staff ratings of disruptive behavior and social/psychological functioning were significantly improved with a medium treatment effect for the DBSP Total score and the Social/Psychological scale, and a small effect for the Disruptive Behavior scale.

\section{Post-treatment outcome with PP analysis}

A similar pattern of results was found when a PP analysis was conducted with the treatment effect increasing for all significant outcomes, now ranging between medium and large effect (see Table 4).

\section{Discussion}

This study aimed to investigate the completion rate and effectiveness of the R\&R2MHP program when delivered to high risk MDOs with SMI. We found a group completion rate of $80 \%$; the $20 \%$ dropout rate is considerably lower than the $50 \%$ dropout rate reported by Cullen et al. [46] for delivery of the 36-session $R \& R$ program to MDOs. Hence the revisions that were made in $R \& R 2$ seem to have made the program more responsive to the needs of this population who are a complex group of 
Table 3 Post-treatment ITT outcome data comparing R\&R2MHP and TAU conditions

\begin{tabular}{|c|c|c|c|c|c|}
\hline & \multicolumn{2}{|c|}{ Baseline (Time 1) } & \multicolumn{2}{|c|}{ Post-treatment (Time 2) } & \multirow{3}{*}{$\begin{array}{c}\text { ITT Time } 2 \text { outcome } \\
\text { F-value } \\
\text { (Cohen's } d \text { ) }\end{array}$} \\
\hline & $\mathrm{R} \& \mathrm{R} 2 \mathrm{MHP}(\mathrm{N}=30)$ & TAU $(\mathrm{N}=29)$ & $\mathrm{R} \& \mathrm{R} 2 \mathrm{MHP}(\mathrm{N}=30)$ & TAU $(\mathrm{N}=29)$ & \\
\hline & Mean (SD) & Mean (SD) & Mean (SD) & Mean (SD) & \\
\hline MVQ Total & $15.83(12.02)$ & $18.13(13.45)$ & $14.60(11.22)$ & $21.34(14.67)$ & $6.26(.52)^{*}$ \\
\hline Machismo scale & $8.60(9.75)$ & 10.79 (11.03) & $8.00(9.21)$ & $12.86(12.05)$ & $4.33(.45)^{*}$ \\
\hline Acceptance scale & $7.23(3.48)$ & $7.34(3.88)$ & $6.60(3.12)$ & $8.48(3.97)$ & $7.62(.53)^{* *}$ \\
\hline NAS-PI total & $77.27(16.78)$ & 79.24 (15.74) & $80.00(16.02)$ & 81.00 (18.45) & 0.01 \\
\hline Cognitive domain & $27.90(6.39)$ & $28.07(5.32)$ & $28.33(5.27)$ & $28.59(5.80)$ & 0.02 \\
\hline Arousal domain & $24.60(6.03)$ & $25.52(6.12)$ & $26.47(7.07)$ & $26.28(6.63)$ & 0.38 \\
\hline Behavior domain & $24.77(5.91)$ & $25.66(6.19)$ & $25.20(5.91)$ & $26.14(7.30)$ & 0.06 \\
\hline WAYS total & $46.90(22.43)$ & $42.83(24.86)$ & $61.50(23.31)$ & $33.41(24.04)$ & $35.56(1.19)^{* * *}$ \\
\hline Confrontive coping & $5.90(3.22)$ & $5.69(3.26)$ & $7.97(3.77)$ & $4.83(3.26)$ & $15.15(0.89)^{* * *}$ \\
\hline Distancing coping & 7.07 (3.59) & $6.21(3.60)$ & $8.93(4.22)$ & $4.45(3.58)$ & $20.42(1.14)^{* * *}$ \\
\hline Self-controlling coping & $5.13(2.53)$ & $3.83(2.56)$ & $6.33(2.89)$ & $3.24(2.84)$ & $13.20(1.08)^{* * *}$ \\
\hline Seeking social support coping & $8.97(7.92)$ & $8.00(5.70)$ & $11.57(7.10)$ & $6.31(4.80)$ & $18.74(0.87)^{* * *}$ \\
\hline Accepting responsibility coping & $3.37(2.34)$ & $2.31(2.59)$ & $3.90(2.50)$ & $1.59(2.38)$ & $10.40(0.95)^{* *}$ \\
\hline Escape-avoidance coping & $4.27(3.23)$ & $4.38(3.91)$ & $6.50(3.03)$ & $4.93(3.57)$ & $9.73(0.47)^{* *}$ \\
\hline Planful problem-solving coping & $6.50(3.62)$ & 7.69 (6.99) & $8.67(3.35)$ & $4.59(4.20)$ & $24.05(1.07)^{* * *}$ \\
\hline Positive reappraisal coping & $5.70(4.11)$ & $4.72(4.38)$ & $7.63(4.63)$ & $3.45(4.35)$ & $15.98(0.93)^{* * *}$ \\
\hline SPSI-RS total & $12.39(3.36)$ & $11.57(2.49)$ & $12.95(3.32)$ & $11.01(3.84)$ & 3.07 \\
\hline Positive problem orientation & $11.20(5.09)$ & $9.41(5.07)$ & $12.13(5.28)$ & $7.86(5.34)$ & $10.73(0.80)^{* *}$ \\
\hline Negative problem orientation & $6.43(4.98)$ & $6.41(4.38)$ & $6.00(3.92)$ & $5.59(4.35)$ & 0.22 \\
\hline Rational problem solving & $10.50(4.82)$ & $8.14(4.99)$ & $10.80(5.62)$ & $6.17(5.31)$ & $6.72(0.85)^{*}$ \\
\hline Impulsivity/carelessness & $7.13(4.88)$ & $7.34(5.13)$ & $6.70(4.34)$ & $5.72(5.21)$ & 1.20 \\
\hline Avoidance style & $6.17(4.32)$ & $5.90(4.19)$ & $5.57(3.86)$ & $4.00(3.35)$ & $4.20(0.43)^{*}$ \\
\hline DBSP total & $35.69(16.12)$ & $39.00(12.51)$ & $31.31(12.96)$ & $41.41(13.34)$ & $11.86(0.77)^{* *}$ \\
\hline Disruptive behavior & $16.88(10.43)$ & $18.44(10.04)$ & $13.81(8.11)$ & $18.15(9.76)$ & $5.20(0.48)^{*}$ \\
\hline Social and psychological & $18.81(7.77)$ & $20.56(6.89)$ & $17.50(7.93)$ & $23.37(6.93)$ & $8.82(0.79)^{* *}$ \\
\hline
\end{tabular}

${ }^{*} \mathrm{p}<.05,{ }^{* *} \mathrm{p}<.01,{ }^{* * *} \mathrm{p}<.001$.

offenders, presenting with severe mental illness and/or disorder, high rates of comorbidity, substance misuse, entrenched antisocial attitudes and rigid cognitive styles. The reduction in duration of the program together with the supplementation of a mentor, who provides individual sessions that aim to transfer skills from the group setting into daily activities, are likely to be important factors in limiting dropout [41].

These findings are consistent with results from a study evaluating the feasibility of R\&R2MHP in low and medium secure settings, reporting a completion rate of $78 \%$ [43]. Cullen et al. caution that treatment dropout may be associated with a high risk status [35], suggesting that completion rates may be inflated by the inclusion of lower risk patients who are more advanced in the rehabilitation pathway. The low dropout rates observed in the current study, together with the $76 \%$ completion rate reported in a study of R\&R2 delivered to high risk patients with a primary diagnosis of personality disorder [45], suggest that the revised program is feasible for treating patients at all levels of secure care.

It is essential to minimise dropout from OBPs as research has indicated that those who do not complete treatment display higher rates of re-offending compared to those who do not engage in treatment at all [36]. Conversely, those who complete treatment programs have lower reconviction rates compared to those who do not complete treatment [38]. In the current study, group non-completers were perceived by staff to be more disruptive and have more social problems than those who went on to complete the group. Their disruptive behaviour and social problems may make their ability to cope with group sessions more difficult and increase the likelihood of dropout.

The second aim of the current study was to evaluate the effectiveness of the program in high risk MDOs with 
Table 4 Post-treatment PP outcome data comparing R\&R2MHP and TAU conditions

\begin{tabular}{|c|c|c|c|c|c|}
\hline & \multicolumn{2}{|c|}{ Baseline (Time 1) } & \multicolumn{2}{|c|}{ Post-treatment (Time 2) } & \multirow{3}{*}{$\begin{array}{l}\text { PP Time } 2 \text { outcome } \\
\text { F-value } \\
\text { (Cohen's } d \text { ) }\end{array}$} \\
\hline & R\&R2MHP (N = 28) & TAU $(\mathrm{N}=20)$ & R\&R2MHP (N = 28) & $\overline{T A U}(\mathrm{~N}=20)$ & \\
\hline & Mean (SD) & Mean (SD) & Mean (SD) & Mean (SD) & \\
\hline MVQ Total & $15.83(12.02)$ & $18.13(13.45)$ & $13.42(10.52)$ & $21.75(15.56)$ & $6.98(0.63)^{*}$ \\
\hline Machismo scale & $8.60(9.75)$ & 10.79 (11.03) & $7.08(8.66)$ & $12.95(13.26)$ & $4.90(0.52)^{*}$ \\
\hline Acceptance scale & $7.23(3.48)$ & $7.34(3.88)$ & $6.35(3.12)$ & $8.80(3.53)$ & $8.75(0.74)^{* *}$ \\
\hline NAS-PI total & $77.27(16.78)$ & $79.24(15.74)$ & $79.12(15.63)$ & $79.25(19.75)$ & 0.00 \\
\hline Cognitive domain & $27.90(6.39)$ & $28.07(5.32)$ & $28.00(5.41)$ & $28.35(6.47)$ & 0.03 \\
\hline Arousal domain & $24.60(6.03)$ & $25.52(6.12)$ & $26.31(7.11)$ & $25.45(6.80)$ & 0.26 \\
\hline Behavior domain & $24.77(5.91)$ & $25.66(6.19)$ & $24.81(5.44)$ & $25.45(7.59)$ & 0.09 \\
\hline WAYS total & $46.90(22.43)$ & $42.83(24.86)$ & $62.08(22.66)$ & $25.65(18.64)$ & $44.83(1.76)^{* * *}$ \\
\hline Confrontive coping & $5.90(3.22)$ & $5.69(3.26)$ & $8.08(4.00)$ & $4.00(2.71)$ & $16.13(1.19)^{* * *}$ \\
\hline Distancing coping & $7.07(3.59)$ & $6.21(3.60)$ & $9.00(4.37)$ & $3.40(3.32)$ & $23.32(1.44)^{* * *}$ \\
\hline Self-controlling coping & $5.13(2.53)$ & $3.83(2.56)$ & $6.65(2.87)$ & $2.55(2.72)$ & $16.56(1.47)^{* * *}$ \\
\hline Seeking social support coping & $8.97(7.92)$ & $8.00(5.70)$ & $10.81(4.32)$ & $4.85(4.08)$ & $26.54(1.42)^{* * *}$ \\
\hline Accepting responsibility coping & $3.37(2.34)$ & $2.31(2.59)$ & $4.12(2.36)$ & $.95(1.88)$ & $18.51(1.49)^{* * *}$ \\
\hline Escape-avoidance coping & $4.27(3.23)$ & $4.38(3.91)$ & $6.69(3.07)$ & $4.60(2.37)$ & $8.23(0.76)^{* *}$ \\
\hline Planful problem-solving coping & $6.50(3.62)$ & $7.69(6.99)$ & $8.85(3.51)$ & $3.45(3.38)$ & $34.59(1.57)^{* * *}$ \\
\hline Positive reappraisal coping & $5.70(4.11)$ & $4.72(4.38)$ & $7.88(4.68)$ & $1.80(3.22)$ & $21.33(1.51)^{* * *}$ \\
\hline SPSI-RS total & $12.39(3.36)$ & $11.57(2.49)$ & $12.91(3.26)$ & $10.37(4.05)$ & 3.94 \\
\hline Positive problem orientation & $11.20(5.09)$ & $9.41(5.07)$ & $11.96(5.40)$ & $6.65(4.32)$ & $13.24(1.09)^{* * *}$ \\
\hline Negative problem orientation & $6.43(4.98)$ & $6.41(4.38)$ & $6.12(3.84)$ & $5.70(4.74)$ & 0.26 \\
\hline Rational problem-solving & $10.50(4.82)$ & $8.14(4.99)$ & $10.50(5.95)$ & $5.05(4.89)$ & $8.25(1.00)^{* *}$ \\
\hline Impulsivity/carelessness & $7.13(4.88)$ & $7.34(5.13)$ & $6.42(4.34)$ & $5.65(5.44)$ & 1.08 \\
\hline Avoidance style & $6.17(4.32)$ & $5.90(4.19)$ & $5.46(3.47)$ & $3.15(2.68)$ & $8.33(0.75)^{* *}$ \\
\hline DBSP total† & $35.69(16.12)$ & $39.00(12.51)$ & $28.46(9.11)$ & 41.06 (13.99) & $13.65(1.07)^{* * *}$ \\
\hline Disruptive behaviort & $16.88(10.43)$ & $18.44(10.04)$ & $11.46(2.96)$ & $17.06(9.59)$ & $8.14(0.79)^{* *}$ \\
\hline Social and psychologicalt & $18.81(7.77)$ & $20.56(6.89)$ & $17.00(8.77)$ & $24.18(7.90)$ & $7.16(0.86)^{*}$ \\
\hline
\end{tabular}

${ }^{*} \mathrm{p}<.05,{ }^{* *} \mathrm{p}<.01,{ }^{* * *} \mathrm{p}<.001$.

$+\mathrm{R} \& \mathrm{R} 2 \mathrm{MHP} N=26$ and TAU $\mathrm{N}=1$.

SMI. As hypothesised, significant treatment effects were found at outcome for self-reported violent attitudes, coping processes and social problem-solving and for staff rated behavior on wards. A conservative ITT analysis found small to large treatment effects all of which improved (to medium and large) when a PP analysis was conducted.

As R\&R2MHP's primary aim is to reduce violent thinking and behavior, the present study used the self-report MVQ as a primary outcome measure to assess attitudes towards violence. Consistent with the findings of previous studies $[43,45]$ a significant treatment effect was found for violent attitudes. This was endorsed by staff ratings of behavior on the wards indicating a significant reduction in social difficulties and disruptive behavior.

By contrast, Young, Hopkin et al. [45] reported significant results at outcome for NAS-PI anger cognitions in offenders with a primary diagnosis of personality disorder which were not supported by the current study or by Rees-Jones et al. [43], the latter studies including offenders with a history of SMI. Around half of the Young, Hopkin et al. [45] sample had borderline personality disorder suggesting that the measure may be more helpful in assessing emotionally labile patients.

Following treatment with the R\&R2MHP program, an improvement in SPSI-RS problem-solving styles has previously been reported for rational thinking [43] and, using a similar program, for avoidance coping [45]. There was no significant treatment effect for impulsivity/carelessness style or the total problem-solving score, thus not supporting the improvement on these scales reported previously when a similar program was delivered to high risk personality disordered patients [45]. The current study was slightly underpowered which may explain lack of significance for these scales, or alternatively there may be some variability in outcomes for MDO patient populations, even for those detained at the same level of security. 
To our knowledge, this is the first time that a treatment effect has been reported indicating greater positive problem orientation in MDOs (large effect) and the finding indicates that participants in the treatment condition had developed more adaptive and constructive methods of social problem-solving compared with those in the control condition. The results provide further support for the existing empirical base which indicates that adaptive social problem-solving can be learnt, even by individuals who are considered to be high risk recidivists [34].

The largest treatment effect was found with respect to coping processes; all outcomes with the exception of one had a large effect size. In the pilot study, conducted on SMI offenders detained in high and medium secure hospital [44], the WAYS total score was not significant, although the pilot had a small control sample of only twelve patients. The present findings are however consistent with a previous study which assessed coping processes using a similar construct, the Coping Responses Inventory, and reported small to large effects in an evaluation of the longer 36-session R\&R program [60]. The present findings indicate that the R\&R2MHP program has a substantially significant effect on coping processes. The increase in confrontive coping is interesting and may not necessarily mean that patients take a more aggressive position, but are more prepared to assert themselves and have confidence to confront and deal with interpersonal problems. Similarly, the increase in distancing and avoidance coping may reflect that patients engage in techniques that help them to walk away from situations that are escalating and/ or anger provoking.

The study has several limitations. Patients were referred as appropriate for the group treatment by their clinical team. Those who were not referred and the reasons for exclusion were not recorded. The largest exclusion categories were likely to be for reasons of mental instability and risk of violence to the researchers, thus reducing the generalisability of the study findings. It was slightly underpowered which may have resulted in lower effect sizes being identified. It has a quasi-experimental controlled design and group allocation was not randomized, thus in order to control for variance at baseline ANCOVA was used with baseline Time 1 scores covarying for the dependent outcome scores and a more conservative ITT analysis selected as the primary analysis.

A randomised methodology was not applied for pragmatic reasons because clinical teams did not wish to unnecessarily delay treatment. Whilst differences in baseline characteristics were not seen among those in the two arms of the trial, numbers were small and it is possible that other factors that influence outcome but were not measured differed between them. Given that those who received active treatment were the first to be referred, it is possible that they were more motivated to change their behavior. Missing data is common for a MDO sample, however we were unable to collect a substantial amount of informant Time 2 data due to high levels of staff turnover on wards. To avoid this in future, it is suggested that informant data is obtained with collective input from the clinical team, i.e. at ward round meetings. A record review of behavior on the ward (i.e. a record of critical incidents) was deemed unhelpful as previous review of such records has indicated a low baseline of incidents, with most patients having no incidents formally recorded. Future research should consider using a prospective assessment of aggression, such as the Staff Observation Aggression Scale Revised (SOAS-R0) [61], to obtain a record of every day, including minor, incidents.

The findings contribute to the growing evidence for treatment using R\&R2MHP in different populations, however findings seem to differ between samples with differing patient characteristics and levels of security. Other characteristics that may influence outcome include IQ [62], self-esteem [63] impulsivity [64,65] and psychopathy [35]. It would be helpful if objective predictors of treatment outcome could be established, as this would mean that services could identify those who are likely to benefit from psychological interventions and those who require preparatory precursor treatments [66]. Outcome measures were administered by researchers who had not been involved in the delivery of the intervention, nevertheless a positive bias may still be present. Outcome measures consisted purely of clinical indicators. In particular, future research should include a cost-effective component in order that health economic outcomes can be assessed. There was no post-group follow up; other studies have reported an increase in treatment effects at 3-month follow-up, indicating that the program has a sustained influence over time $[43,67]$. Future research should use a randomized controlled design with follow-up data that includes objective assessments and reconviction data. It is of note that a metaanalysis of the original $R \& R$ programme, on which the revised $R \& R 2$ is based, found reductions in re-offending [34].

\section{Conclusions}

The results of the present study support the use of group based cognitive-behavioral skills interventions with MDOs, and the R\&R2MHP program has the advantage of being a manualised program which maximises program integrity. In spite of the aforesaid limitations, results from the present study contribute to its accruing evidence-base. Significant improvements were found in violent attitudes, coping processes and social problem-solving, and were endorsed by ratings of ward behavior. We are living in an era that demands cost-effective solutions for treating people with mental illness and group-work programs are a recognised target for development (The Sainsbury Centre for Mental Health, 2008). R\&R2MHP is nearly half the 
duration of its predecessor and seems to be equally effective, hence representing a cost-effective program.

\section{Abbreviations}

DBSP: Disruptive behavior and social problem scale; MDO: Mentally disordered offender; MVQ: Maudsley violence questionnaire; NAS-PI: Novaco anger scale and provocation inventory; OBP: Offending behavior programs; R\&R: Reasoning and rehabilitation; R\&R2 MHP: Reasoning and rehabilitation 2 for youths and adults with mental health problems; SMI: Severe mental illness; SPSI-RS: Social problem solving scale revised, short version; TAU: Treatment as usual; WAYS: Ways of coping scale.

\section{Competing interests}

SY is a consultant for the Cognitive Centre of Canada and co-author of R\&R2MHP. The remaining authors declare they have no competing interests.

\section{Authors' contributions}

SY, GG and DP contributed to the study design and management of the project, assisted by $A D$ and $G H$. SY provided training in R\&R2MHP. AD, GH and VY completed data collection and input. VY conducted the statistical analysis and completed a first draft of the manuscript. SY, GG and DP made revisions and edits to subsequent drafts. All authors have read and approved the manuscript.

\section{Author details}

'Department of Forensic and Neurodevelopmental Sciences, PO23, King's College London, Institute of Psychiatry, De Crespigny Park, London SE5 8AF, UK. ${ }^{2}$ Broadmoor Hospital, Crowthorne, UK. ${ }^{3}$ Department of Psychology, PO77, King's College London, Henry Wellcome Building, De Crespigny Park, London SE5 8AF, UK.

Received: 8 February 2013 Accepted: 10 October 2013

Published: 18 October 2013

\section{References}

1. Hodgins S: Mental disorder, intellectual deficiency, and crime: evidence from a birth cohort. Arch Gen Psychiatry 1992, 49:476-483.

2. Wallace $C$, Mullen PE, Burgess P: Criminal offending in schizophrenia over a 25 -year period marked by deinstitutionalization and increasing prevalence of co-morbid substance use disorders. Am J Psychiatr 2004, 161:716-727.

3. Brennan A, Mednick SA, Hodgins S: Major mental disorders and criminal violence in a Danish birth cohort. Arch Gen Psychiatry 2000, 5:494-500.

4. Fazel $S$, Danesh J: Serious mental disorder in 23000 prisoners: a systematic review of 62 surveys. Lancet 2002, 359:545-550.

5. Swanson JW, Swartz MS, Van Dorn RA, Elbogen EB, Wagner HR, Rosenheck RA, Stroup TS, McEvoy JP, Lieberman JA: A national study of violent behavior in persons with schizophrenia. Arch Gen Psychiatry 2006, 63:490-496.

6. Hodgins S, Alderton J, Cree A, Aboud A, Mak T: Aggressive behavior, victimisation and crime among severely mentally ill patients requiring hospitalisation. Br J Psychiatry 2007, 191:343-350.

7. Walsh E, Buchanan A, Fahy T: Violence and schizophrenia: examining the evidence. Br J Psychiatry 2002, 180:490-495.

8. Swanson JW, Borum R, Swartz M, Hiday V: Violent behavior preceding hospitalisation among persons with severe mental illness. Law Hum Behav 1999, 23:185-204.

9. Rutherford M, Duggan S: Forensic mental health services: facts and figures on current provision, The Sainsbury centre for mental health, forensic fact-file. 2007. http://www.centreformentalhealth.org.uk/pdfs/scmh_forensic_factfile_2007.pdf.

10. Bonta J, Law M, Hanson K: The prediction of criminal and violent recidivism among mentally disordered offenders: a meta-analysis. Psychol Bull 1998, 123:123-142.

11. Gray NS, Snowden RJ, MacCulloch S, Phillips H, Taylor J, MacCulloch MJ: Relative efficacy of criminological, clinical, and personality measures of future risk of offending in mentally disordered offenders: a comparative study of HCR-20, PCL:SV, and OGRS. J Consult Clin Psychology 2004, 72:523-530.

12. Kroner DG, Mills JF, Morgan RD: Underreporting of crime-related content and the prediction of criminal recidivism among violent offenders. Psychol Serv 2007, 4:85-95.
13. Morgan RD, Fisher WH, Duan N, Mandracchia JT, Murray D: Prevalence of criminal thinking among state prison inmates with serious mental illness. Law Hum Behav 2010, 34:324-336.

14. D'Zurilla TJ, Nezu AM, Maydeu-Olivares A: A Social Problem-Solving InventoryRevised (SPSI-R). North Tonawanda: NY: Technical Manual. Multi-Health Systems; 2002

15. Biggam FH, Power KG: Social problem-solving skills and psychological distress among incarcerated young offenders: the issue of bullying and victimization. Cogn Ther Res 1999, 23:307-326.

16. Ingram JC, Dixon DN, Glover JA: Problem solving as a function of race and incarceration. J Soc Psychol 1983, 120:83-90.

17. McMurran M, Egan V, Richardson C, Ahmadim S: Social problem-solving in mentally disordered offenders: a brief report. Crim Behav Ment Health 1999, 9:315-322.

18. Gendreau P, Goggin C, Smith P: Is the PCL-R really the "unparalleled" measure of offender risk? a lesson in knowledge cumulation. Crim Justice Behav 2002, 29:397-426.

19. Gendreau P, Little T, Goggin C: A meta-analysis of the predictors of adult offender recidivism: what works! Criminology 1996, 34:575-608

20. Simourd DJ, Olver ME: The future of criminal attitudes research and practice. Crim Justice Behav 2002, 29:427-446.

21. Barriga $A Q$, Gibbs JC, Potter GB, Liau AK: How I think (HIT) questionnaire manual. Champaign: Research Press; 2001.

22. Best D, Day E, Campbell A, Flynn PM, Simpson DD: Relationship between drug treatment engagement and criminal thinking style among drugusing offenders. Eur Addict Res 2009, 15:71-77.

23. Brezina T: Anger, attitudes, and aggressive behavior: exploring the affective and cognitive foundations of angry aggression. $J$ Contemp Crim Justice 2010, 26:186-203.

24. Howells K: Anger and its links to violent offending. Psychiatry Psychol Law 2004, 11:189-196.

25. Zamble E, Porporino F: Coping, imprisonment, and rehabilitation: some data and their implications. Crim Justice Behav 1990, 17:53-70.

26. Taylor PJ, Leese M, Williams D, Butwell M, Daly R, Larkin E: Mental disorder and violence: a special (high security) hospital study. Br J Psychiatry 1998, 172:218-226.

27. Thomas S, Leese M, Dolan M, Harty M, Shaw J, Middleton H, Carlisle J, Davies $L$, Thornicroft $G$, Appleby $L$ : The individual needs of patients in high secure psychiatric hospitals in England. J Forensic Psychiatry Psychol 2004, 15:222-243.

28. Martinson R: What works? questions and answers about prison reform. Public Interest 1974, 35:22-54.

29. McGuire J: What works: reducing reoffending: guidelines from research and practice. Wiley series in offender rehabilitation. Oxford: John Wiley \& Sons; 1995.

30. Blud L, Travers R, Nugent F, Thorton D: Accreditation of offending behaviour programmes in HM prison service: 'what Works' in practice. Leg Criminol Psychol 2003, 8:69-81.

31. Clark DA: Theory manual for enhanced thinking skills: prepared for the joint prison accreditation panel. London: Home Office; 2000.

32. Ross RR, Fabiano EA, Ewles CD: Reasoning and rehabilitation. Int $J$ Offender Ther Comp Criminol 1988, 32:29-35.

33. Landenberger NA, Lipsey MW: The positive effects of cognitive-behavioral programs for offenders: a meta-analysis of factors associated with effective treatment. J Exp Criminol 2005, 1:451-476.

34. Tong LS, Farrington DP: How effective is the "reasoning and rehabilitation" program in reducing reoffending? a meta-analysis of evaluations in four countries. Psychol Crime and Law 2006, 12:3-24

35. Cullen AE, Soria C, Clarke AY, Dean K, Fahy T: Factors predicting dropout from the reasoning and rehabilitation program with mentally disordered offenders. Crim Justice Behav 2011, 38:217-230.

36. McMurran M, Theodosi E: Is treatment non-completion associated with increased reconviction over no treatment. Psychol Crime Law 2007, 13:333-343

37. Wormith JS, Olver ME: Offender treatment attrition and its relationship with risk, responsivity, and recidivism. Crim Justice Behav 2002, 29:447-471

38. Hollin CR, McGuire J, Hounsome JC, Hatcher RM, Bilby CAL, Palmer EJ: Cognitive skills behavior programs for offenders in the community: a reconviction analysis. Crim Justice Behav 2008, 35:269-283.

39. Andrews DA, Bonta J: Rehabilitating Criminal Justice Policy and Practice. Psychol Publ Pol Law 2010, 16:39-55. 
40. Young SJ, RosS RR: R\&R2 for youths and adults with mental health problems: a prosocial competence training program. Ottawa: Cognitive Centre of Canada; 2007.

41. Hollin CR, Palmer E: Cognitive skills program for offenders. Psychol Crime Law 2009, 15:147-164.

42. McGurk SR, Twamley EW, Sitzer DI, McHugo GJ, Mueser KT: A meta-analysis of cognitive remediation in schizophrenia. Am J Psychiatry 2007, 164:1791-1802.

43. Rees-Jones A, Gudjonsson G, Young S: A multi-site controlled trial of a cognitive skills program for mentally disordered offenders. BMC Psychiatry 2012, 12:1-11.

44. Young S, Chick K, Gudjonsson G: A preliminary evaluation of reasoning and rehabilitation 2 in mentally disordered offenders (R\&R2M) across two secure forensic settings in the United Kingdom. J Forensic Psychiatry Psychol 2010, 21:336-349.

45. Young S, Hopkin G, Perkins D, Farr C, Doidge A, Gudjonsson GH: A controlled trial of a cognitive skills program for personality disordered offenders. J Atten Disord, 17:598-607.

46. Cullen AE, Clarke AY, Kuipers E, Hodgins S, Dean K, Fahy T: A multi-site randomized controlled trial of a cognitive skills program for male mentally disordered offenders: social-cognitive outcomes. Psychol Med 2012, 42:557-569.

47. Haddock G, McCarron J, Tarrier N, Faragher EB: Scales to measure dimensions of hallucinations and delusions: the psychotic symptom rating scales (PSYRATS). Psychol Med 1999, 29:879-889.

48. Spitzer RL, Williams JBW, Gibbon M, First MB: Structured clinician interview for DSM-III-R axis II disorders (SCID-II). Washington: American Psychiatric Press; 1990.

49. Walker JS: The maudsley violence questionnaire: initial validation and reliability. Personal Individ Differ 2005, 38:187-201.

50. Novaco RW: The novaco anger scale and provocation inventory. Los Angeles: Western Psychological Services; 2003.

51. Novaco RW, Taylor JL: Assessment of anger and aggression in offenders with developmental disabilities. Psychol Assess 2004, 16:42-50.

52. Mills JF, Kroner DG, Forth AE: Novaco anger scale: reliability and validity within an adult criminal sample. Assessment 1998, 5:327-248.

53. Monahan J, Steadman H, Silver E, Appelbaum P, Robbins P, Mulvey E, Banks S: Rethinking risk assessment: the MacArthur study of mental disorder and violence. New York: Oxford University Press; 2001.

54. Jones JP, Thomas-Peter BA, Trout A: Normative data for the novaco anger scale from a non-clinical sample and implications for clinical use. Br J Clin Psychol 1999, 38:417-424.

55. Lazarus R, Folkman S: Stress, appraisal and coping. New York: Springer; 1984

56. Folkman S, Lazarus RS: If it changes it must be a process: study of emotion and coping during three stages of a college examination. J Pers Soc Psychol 1985, 48:150-170.

57. D'Zurilla TJ, Nezu AM, Maydeu-Olivares A: A social problem-solving InventoryRevised (SPSI-R): technical manual. Multi-Health Systems: North Tonawanda; 2002.

58. Young S, Gudjonsson G, Ball S, Lam J: Attention deficit hyperactivity disorder in personality disordered offenders and the association with disruptive behavioural problems. J Forensic Psychiatry Psychol 2003, 14:491-505.

59. Prince M, Stewart R, Ford T, Hotopf M: Practical psychiatric epidemiology. Oxford: Oxford Medical Publications; 2003.

60. Clarke AY, Cullen AE, Walwyn R, Fahy T: A quasi-experimental pilot study of the reasoning and rehabilitation program with mentally disordered offenders. J Forensic Psychiatry Psychol 2010, 21:490-500.

61. Nijman HL, Muris P, Merckelbach HG, Palmsteir T, Wistedt B, Vos AM, Rixtel AV Allertz W: The staff observation aggression scale- revised (SOAS-R). Aggress Behav 1999, 25:197-209.

62. McMurran M, Huband N, Duggan C: A comparison of treatment completers and non-completers of an in-patient treatment program for male personality-disordered offenders. Psychol Psychother Theory Res Pract 2008, 81:193-198.

63. Laithwaite HM, Gumley A, Benn A, Scott E, Downey K, Black K, McEwen S: Self esteem and psychosis: a pilot study investigating the effectiveness of a self-esteem program on the self-esteem and positive symptomatology of mentally disordered offenders. Behav Cogn Psychother 2007, 35:569-577.

64. Mak AS: Psychosocial control characteristics of delinquents and nondelinquents. Crim Justice Behav 1991, 18:297-303.

65. Eysenck SB, McGurk BJ: Impulsiveness and venturesomeness in a detention centre population. Psychol Rep 1980, 47:1299-1306.

66. Gudjonsson G, Young S: The role and scope of forensic clinical psychology in secure unit provision: a proposed service model for psychological therapies. J Forensic Psychiatry Psychol 2007, 18:534-556.

67. Emilsson B, Gudjonsson G, Sigurdsson JF, Baldursson G, Einarsson E, Olafsdottir H, Young S: Cognitive behavior therapy in medication-treated adults with ADHD and persistent symptoms: a randomized controlled trial. BMC Psychiatry 2011, 11:1-10.

doi:10.1186/1471-244X-13-267

Cite this article as: Yip et al:: A non-randomised controlled trial of the R\&R2MHP cognitive skills program in high risk male offenders with severe mental illness. BMC Psychiatry 2013 13:267.

\section{Submit your next manuscript to BioMed Central and take full advantage of:}

- Convenient online submission

- Thorough peer review

- No space constraints or color figure charges

- Immediate publication on acceptance

- Inclusion in PubMed, CAS, Scopus and Google Scholar

- Research which is freely available for redistribution 